\title{
Basic science: (December 2007)
}

1. Antoniou AC, Sinilnikova OM, Simard J, Leone $M$, Dumont M, Neuhausen SL, Struewing JP, Stoppa-Lyonnet D, Barjhoux L, Hughes DJ, Coupier I, Belotti M, Lasset C, Rebbeck TR, Wagner T, Lynch HT, Domchek SM, Nathanson KL, Garber JE, Weitzel J, Narod SA, Tomlinson G, Olopade OI, Godwin A, Isaacs C, Jakubowska A, Lubinski J, Gronwald J, Gorski B, Byrski T, Huzarski T, Peock S, Cook M, Baynes C, Murray A, Rogers M, Daly PA, Dorkins $H$, Schmutzler RK, Versmold B, Engel C, Meindl A, Arnold N, Niederacher D, Deissler H, Spurdle $A B$, Chen XQ, Waddell N, Cloonan N, Kirchhoff T, Offit K, Friedman E, Kaufmann B, Laitman Y, Galore G, Rennert G, Lejbkowicz F, Raskin L, Andrulis IL, Ilyushik E, Ozcelik H, Devilee P, Vreeswijk MPG, Greene MH, Prindiville SA, Osorio A, Benitez J, Zikan M, Szabo Cl, Kilpivaara O, Nevanlinna H, Hamann U, Durocher F, Arason A, Couch FJ, Easton DF, ChenevixTrench G. RADS1 135G -> C modifies breast cancer risk among BRCA2 mutation carriers: results from a combined analysis of 19 studies. Am J Hum Genet 2007; 81: 1186-1200.

2. Bayliss J, Hilger A, Vishnu P, Diehl K, El Ashry $D$. Reversal of the estrogen receptor-negative phenotype in breast cancer and restoration of antiestrogen response. Clin Cancer Res 2007; 13: 7029-7036.

3. Belguise K, Sonenshein GE. PKC $\Theta$ promotes c-Rel-driven mammary tumorigenesis in mice and humans by repressing estrogen receptor $\alpha$ synthesis. J Clin Invest 2007; 117: 4009-4021.

4. Bell DW, Kim SH, Godwin AK, Schiripo TA, Harris PL, Haserlat SM, Wahrer DCR, Haiman CA, Daly MB, Niendorf KB, Smith MR, Sgroi DC, Garber JE, Olopade OI, Le Marchand L, Henderson BE, Altshuler D, Haber DA, Freedman ML. Genetic and functional analysis of CHEK2 (CHK2) variants in multiethnic cohorts. Int $J$ Cancer 2007; 121: 2661-2667.

5. Croucher DR, Saunders DN, Stillfried GE, Ranson M. A structural basis for differential cell

BCO/831/2007/JW signalling by PAl-1 and PAl-2 in breast cancer cells. Biochem J 2007; 408: 203-210.

6. Daniel AR, Faivre EJ, Lange CA. Phosphorylation-dependent antagonism of sumoylation derepresses progesterone receptor action in breast cancer cells. Mol Endocrinol 2007; 21: 2890-2906.

7. Dhasarathy A, Kajita M, Wade PA. The transcription factor snail mediates epithelial to mesenchymal transitions by repression of estrogen receptor- $\alpha$. Mol Endocrinol 2007; 21: 2907-2918.

8. Dillon RL, Brown ST, Ling C, Shioda T, Muller WJ. An EGR2/CITED1 transcription factor complex and the 14-3-3 $\sigma$ tumor suppressor are involved in regulating ErbB2 expression in a transgenic-mouse model of human breast cancer. Mol Cell Biol 2007; 27: 8648-8657.

9. Gail MH, Costantino JP, Pee D, Bondy M, Newman L, Selvan M, Anderson GL, Malone KE, Marchbanks PA, McCaskill-Stevens W, Norman SA, Simon MS, Spirtas R, Ursin G, Bernstein $L$. Projecting individualized absolute invasive breast cancer risk in African American women. J Natl Cancer Inst 2007; 99: 1782-1792.

10. Gomez BP, Riggins RB, Shajahan AN, Klimach U, Wang A, Crawford AC, Zhu Y, Zwart A, Wang $M$, Clarke R. Human X-Box binding protein-1 confers both estrogen independence and antiestrogen resistance in breast cancer cell lines. FASEB J 2007; 21: 4013-4027.

11. Gupta GP, Perk J, Acharyya S, de Candia P, Mittal V, Todorova-Manova K, Gerald WL, Brogi E, Benezra R, Massague J. ID genes mediate tumor reinitiation during breast cancer lung metastasis. Proc Natl Acad Sci USA 2007; 104: 19506-19511.

12. Heaphy $C M$, Baumgartner $K B$, Bisoffi $M$, Baumgartner RN, Griffith JK. Telomere DNA content predicts breast cancer-free survival interval. Clin Cancer Res 2007; 13: 7037-7043.

13. Heinonen M, Fagerholm R, Aaltonen K, Kilpivaara O, Aittomaki K, Blomqvist C, Heikkila P, Haglund C, Nevanlinna H, Ristimaki A. Prognostic role of 
HuR in hereditary breast cancer. Clin Cancer Res 2007; 13: 6959-6963.

14. Huang Y, Fernandez SV, Goodwin S, Russo PA, Russo IH, Sutter TR, Russo J. Epithelial to mesenchymal transition in human breast epithelial cells transformed by $17 \beta$-estradiol. Cancer Res 2007; 67: 11147-11157.

15. John EM, Miron A, Gong G, Phipps Al, Felberg A, Li FP, West DW, Whittemore AS. Prevalence of pathogenic BRCA1 mutation carriers in 5 US racial/ethnic groups. JAMA 2007; 298: 2869-2876.

16. John EM, Schwartz GG, Koo J, Wang W, Ingles $S A$. Sun exposure, vitamin $D$ receptor gene polymorphisms, and breast cancer risk in a multiethnic population. Am J Epidemiol 2007; 166: 1409-1419.

17. Lin J, Manson JE, Selhub J, Buring JE, Zhang SMM. Plasma cysteinylglycine levels and breast cancer risk in women. Cancer Res 2007; 67: 11123-11127.

18. MacAusland SG, Hepel JT, Chong FK, Galper SL, Gass JS, Ruthazer R, Wazer DE. An attempt to independently verify the utility of the Van Nuys Prognostic Index for ductal carcinoma in situ. Cancer 2007; 110: 2648-2653.

19. Mori N, Glunde K, Takagi T, Raman V, Bhujwalla ZM. Choline kinase down-regulation increases the effect of 5-fluorouracil in breast cancer cells. Cancer Res 2007; 67: 11284-11290.

20. Naiki-lto A, Asamoto M, Hokaiwado N, Takahashi S, Yamashita H, Tsuda H, Ogawa $\mathrm{K}$, Shirai T. Gpx2 is an overexpressed gene in rat breast cancers induced by three different chemical carcinogens. Cancer Res 2007; 67: 11353-11358.

21. Palmer CP, Mahen R, Schnell E, Djamgoz MBA, Aydar E. Sigma-1 receptors bind cholesterol and remodel lipid rafts in breast cancer cell lines. Cancer Res 2007; 67: 11166-11175.

22. Patocs A, Zhang L, Xu YM, Weber F, Caldes T, Mutter GL, Platzer P, Eng C. Breast-cancer stromal cells with TP53 mutations and nodal metastases. N Engl J Med 2007; 357: 2543-2551.

23. Ruhe JE, Streit S, Hart S, Wong CH, Specht K, Knyazev P, Knyazeva T, Tay LS, Loo HL, Foo P, Wong W, Pok S, Lim SJ, Ong H, Luo M, Ho HV, Peng K, Lee TC, Bezler M, Mann C, Gaertner S,
Hoefler H, lacobelli S, Peter S, Tay A, Brenner $S$, Venkatesh B, Ullrich A. Genetic alterations in the tyrosine kinase transcriptome of human cancer cell lines. Cancer Res 2007; 67: 11368-11376.

24. Sankaran S, Crone DE, Palazzo RE, Parvin JD. Aurora - a kinase regulates breast cancerassociated gene 1 inhibition of centrosomedependent microtubule nucleation. Cancer Res 2007; 67: 11186-11194.

25. Sansone P, Storci G, Tavolari S, Guarnieri T, Giovannini C, Taffurelli M, Ceccarelli C, Santini D, Paterini P, Marcu KB, Chieco P, Bonafe M. IL-6 triggers malignant features in mammospheres from human ductal breast carcinoma and normal mammary gland. J Clin Invest 2007; 117: 3988-4002.

26. Wang H, Teske D, Tess A, Kohlhepp R, Choi Y, Kendziorski C, Moser AR. Identification of novel modifier loci of $\mathrm{Apc}^{\mathrm{Min}}$ affecting mammary tumor development. Cancer Res 2007; 67: 11226-11233.

27. Westbrook L, Manuvakhova M, Kern FG, Estes NR, Ramanathan HN, Thottassery JV. Cks1 regulates cdk 1 expression: a novel role during mitotic entry in breast cancer cells. Cancer Res 2007; 67: 11393-11401.

28. Yokota T, Bui TY, Liu YN, Yi M, Hunt KK, Keyomarsi K. Differential regulation of elafin in normal and tumor-derived mammary epithelial cells is mediated by CCAAT/Enhancer binding protein $\beta$. Cancer Res 2007; 67: 11272-11283.

29. Yokoyama S, Chen CJ, Nguyen T, Shively JE. Role of CEACAM1 isoforms in an in vivo model of mammary morphogenesis: mutational analysis of the cytoplasmic domain of CEACAM1$4 S$ reveals key residues involved in lumen formation. Oncogene 2007; 26: 7637-7646.

30. Zuo T, Liu RH, Zhang HM, Chang X, Liu Y, Wang LZ, Zheng P, Liu Y. FOXP3 is a novel transcriptional repressor for the breast cancer oncogene SKP2. J Clin Invest 2007; 117: 3765-3773.

Prepared by

$R$ Sutherland

Cancer Research Program

Garvan Institute of Medical Research

Darlinghurst, NSW, Australia 